\title{
论 文
}

\section{页岩气藏数值模拟方法评价及选择}

\author{
糜利栋 ${ }^{1,2,3^{*}}$, 姜汉桥 ${ }^{3}$, 胡向阳 ${ }^{1,2}$, 李俊键 $^{3}$, 贾英 ${ }^{1,2}$ \\ 1. 中国石油化工股份有限公司石油勘探开发研究院, 北京 100083; \\ 2. 中国石油化工股份有限公司海相油气藏开发重点实验室, 北京 100083; \\ 3. 中国石油大学(北京)石油工程学院, 北京 102249 \\ *E-mail: cupmld@gmail.com
}

收稿日期：2017-05-20; 接受日期：2017-11-07; 网络版发表日期：2018-04-23

国家重点基础研究发展计划(编号: 2013CB228005)和中国石油化工股份有限公司科技部项目(编号: G5800-17-ZS-KJB004)资助

\begin{abstract}
摘要 复杂的裂缝网络给页岩气藏数值模拟研究带来巨大挑战. 本文利用离散裂缝模型(DFM), 分别建立微尺度 模型和矿场尺度模型，研究了不同基质渗透率和裂缝开度对应条件下，不同类型裂缝对页岩气总产量的贡献率。 研究结果表明: 在量级约为 $\left(10^{-8} \sim 10^{-4}\right) \times 10^{-3} \mu \mathrm{m}^{2}$ 的页岩气藏基质渗透率范围内, 且存在SRV改造区的条件下, 页岩 气产量以 SRV内人工裂缝的贡献为主, SRV内以及 SRV外天然裂缝的贡献量相对较小. 因此, 综合考虑计算复杂度 和计算精度, 可通过构建基质-天然裂缝表观渗透率和连续介质模型的方法表征天然裂缝, 采用离散介质模型中 计算网格少、效率高的离散裂缝网络(DFN)模型表征人工裂缝.
\end{abstract}

关键词 天然裂缝, 人工裂缝, 改造体积, 页岩气藏, 数值模拟方法

\section{1 前言}

从20世纪60年代开始，国内外学者就开始了裂缝 性储层数值模拟的研究工作.但由于裂缝的复杂性和 强非均质性, 研究难度极大, 结果仍不尽如人意. 将裂 缝大尺度平均化、避免单独处理裂缝的“连续介质模 型”虽然计算效率高, 但无法准确描述复杂裂缝网络渗 流特征 ${ }^{[1]}$; 离散裂缝模型(DFM) 将裂缝离散成除裂缝 交点外更多节点的处理方法使得该方法计算量大、处 理复杂缝网能力弱 ${ }^{[2-4]}$; 相比离散裂缝模型，只在裂缝 交点处离散的离散裂缝网络模型(DFN)大幅度降低了 网格数量, 提高了计算效率, 增加了缝网的处理能
力 $^{[5 \sim 10]}$, 但是当存在大量天然裂缝时, 同样会存在计算 量大的问题, 并且DFN只能处理连通裂缝 ${ }^{[7 \sim 9,11]}$. 在前人 研究成果的基础上，按照矿场尺度高效页岩气藏数值 模拟的需要，对页岩储层孔隙结构重新分类. 然后利 用微尺度模型计算不同基质渗透率、不同裂缝开度情 况下, 裂缝和流出边界连通、或不连通时对应的模型 等效渗透率，分析模型等效渗透率与基质渗透率、裂 缝开度以及连通性之间的关系. 基于矿场尺度模型研 究了裂缝开度在 $0.1 \sim 1000 \mu \mathrm{m}$, 基质渗透率在 $(0.0001$ $\sim 0.1) \times 10^{-3} \mu \mathrm{m}^{2}$ 之间变化时，改造体积(SRV，本文提到 的SRV指所有人工裂缝分布的区域)内只有人工裂 缝、SRV内包含天然裂缝、SRV外包含天然裂缝、所

引用格式: 糜利栋, 姜汉桥, 胡向阳, 等. 页岩气藏数值模拟方法评价及选择. 中国科学: 技术科学, 2018, 48: 680-690

Mi L D, Jiang H Q, Hu X Y, et al. Evaluation and selection of numerical simulation methods for shale gas reservoirs (in Chinese). Sci Sin Tech, 2018, 48: 680-690, doi: 10.1360/N092017-00152 
有裂缝均为天然裂缝以及没有裂缝时各模型产量变化 关系，明确了人工裂缝和天然裂缝对页岩气总产量的 贡献率，确定了适用于页岩气藏的数值模拟方法和不 同类型裂缝的合理处理方法.

\section{2 裂缝类型分类}

近几年, 国内外学者对页岩储层孔隙结构进行了 大量研究，但仍没有统一的分类方法 ${ }^{[12 \sim 15]}$. 页岩储层 发育的种类多样、结构复杂、孔径范围极广的复杂孔 隙-微裂缝系统, 再加上人工压裂改造产生的人工裂 缝, 构成了页岩气开发过程中的整个渗流系统. 但是, 由于计算效率和跨尺度渗流等原因, 很难完整地实现 整个渗流系统渗流过程的数值模拟. 所以，为了尽可 能地在矿场尺度页岩气藏数值模拟中表征不同尺度孔 隙中的渗流过程和渗流机理，在前人研究成果的基础 上，按照矿场尺度高效页岩气藏数值模拟的需要，将 页岩储层渗流系统分为基质孔隙渗流系统和裂缝孔隙 渗流系统, 并给出相应渗流特征和渗流过程 ${ }^{[16,17]}$ (表1).

裂缝孔隙又分为人工裂缝和天然裂缝. 在对人工 裂缝和天然裂缝特征 ${ }^{[19,20]}$ 认识的基础上，本文假设“人 工裂缝”包括压裂时产生的新裂缝和经过压裂诱导开 启的天然裂缝, 人工裂缝构成整个SRV区域, 且包含所 有连通裂缝; “天然裂缝”指压裂后仍然无法连通的裂 缝, 包含所有的不连通裂缝 ${ }^{[21]}$.

\section{3 不同裂缝对渗流的影响}

离散介质模型已成为国内外学者用于页岩气数值 模拟研究的重要方法 ${ }^{[4,22,23]}$, 但对于不同类型裂缝对渗 流的贡献率以及不同裂缝合理的表征方法认识不清. 本文首先建立微尺度模型(图1), 通过边界元(BEM)方 法 ${ }^{[24]}$ 求解控制方程, 模拟裂缝性介质中流体流动过程,
计算等效渗透率，研究不同裂缝开度、基质渗透率对 应条件下等效渗透率与基质渗透率和裂缝开度之间的 变化关系. 然后建立矿场尺度模型, 研究不同裂缝开 度、基质渗透率对应条件下，不同类型裂缝对总产量 的贡献率.

\section{1 微尺度模型}

如图1所示, 建立大小为 $1 \mathrm{~cm} \times 1 \mathrm{~cm}$ 的微尺度模型, 其中左边界为流入边界, 压力为 $10 \mathrm{MPa}$, 右边界为流 出边界, 压力为 $5 \mathrm{MPa}$, 上下为无流动边界. 图1(a)为基 础模型, 不包含裂缝, 图1(b)模型中的裂缝和流出边界 连通, 而图1(c)中的裂缝和边界不连通, 采用BEM方法 求解控制方程(详见参考文献[24]). 然后利用Durlofs$\mathrm{ky}^{[25]}$ 提出的等效渗透率计算方法(详见参考文献 [25]) 计算不同模型对应的等效渗透率，分析不同的基质渗 透率 $(0.0001 \sim 0.1) \times 10^{-3} \mu \mathrm{m}^{2}$ 和不同裂缝开度 $(0.05 \sim$ $1000 \mu \mathrm{m})$ 对应条件下等效渗透率与基质渗透率之比 $\left(k_{\mathrm{eq}} / k_{\mathrm{m}}\right)$ 的变化规律.

微尺度模型压力分布如图2所示：图2(a)对应 图1(a)中的基础模型; 图2(b)对应图1(b)中的连通裂缝 模型; 图2(c)对应图1(c)中的不连通裂缝模型. 图2模型 中对应的裂缝开度为 $1 \mu \mathrm{m}$ (利用立方定律计算对应渗 透率为 $83.33 \times 10^{-3} \mu \mathrm{m}^{2}$ ), 基质渗透率为 $0.001 \times 10^{-3} \mu^{2}$. 对比图 2(a) (c), 当基质渗透率等于 $0.001 \times 10^{-3} \mu \mathrm{m}^{2}$ 时, 裂缝影响压力波的传播; 对比图2(b)和(c), 压力分布的 差别主要集中在右边界. 因此, 在此条件下裂缝和流出 边界的连通情况对压力分布影响明显, 由于图2(b)中 裂缝和流出边界连通, 所以压力波比图2(c)传播得 更快.

裂缝开度一定时, $k_{\mathrm{eq}} / k_{\mathrm{m}}$ 和基质渗透率关系曲线如 图3(a)所示. 当裂缝开度为 $0.4 \mu \mathrm{m}$ 时，随着基质渗透率 的增加, 裂缝对等效渗透率的贡献逐渐减小, 当基质渗 透率超过 $0.001 \times 10^{-3} \mu \mathrm{m}^{2}$ 时, 裂缝对渗流能力的贡献不

表 1 孔隙类型分类及其渗流特征 ${ }^{[18]}$

\begin{tabular}{|c|c|c|c|c|}
\hline & & 介质尺度 & 渗流过程 & 渗流特征 \\
\hline \multirow{3}{*}{ 基质孔隙 } & 有机质孔隙 & 分子 & 干酪根 $\rightarrow$ 孔隙表面 & 扩散效应 \\
\hline & 孔隙表面 & 纳米 & 孔隙表面 $\rightarrow$ 微米-纳米孔隙 & 吸附-解吸附效应 \\
\hline & 微米-纳米孔隙 & 微观 & 微米-纳米孔隙一裂缝/井 & 扩散、滑脱效应 \\
\hline \multirow{2}{*}{ 裂缝孔隙 } & 天然裂缝 & 介观 & 天然裂缝～$\rightarrow$ 人裂缝 & 裂缝渗流 \\
\hline & 人工裂缝 & 宏观 & 人工裂缝 $\rightarrow$ 井底 & 裂缝渗流 \\
\hline
\end{tabular}



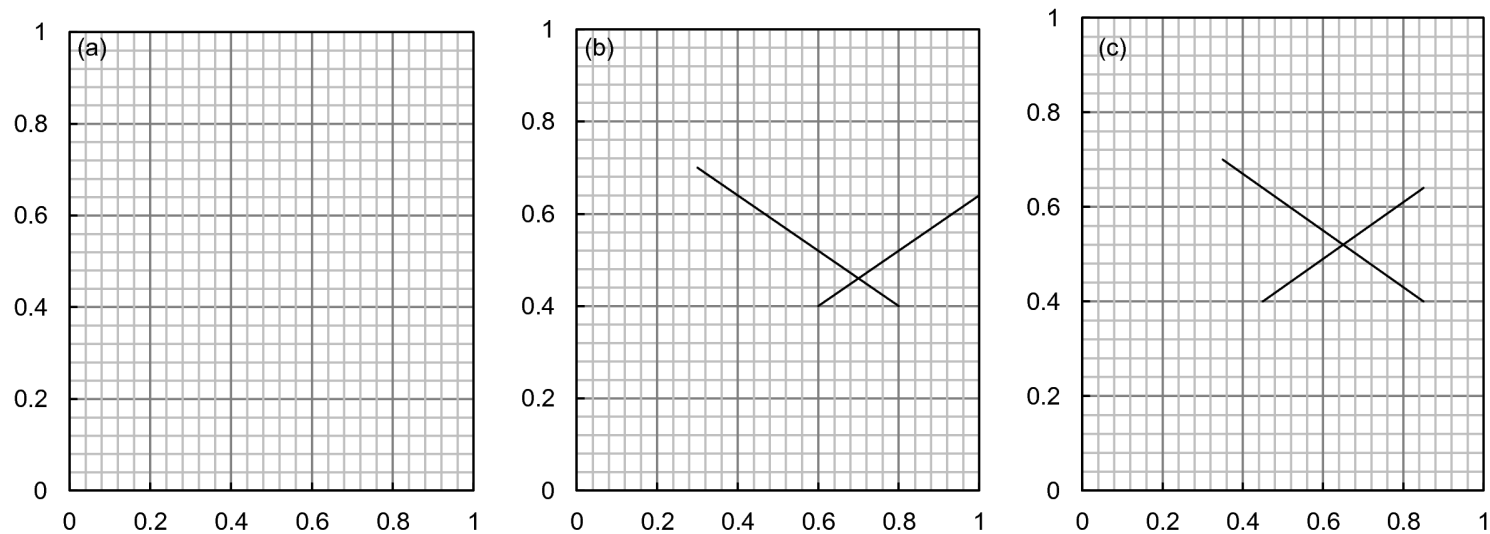

图 1 微尺度模型示意图. (a) 模型I-基础模型; (b) 模型II-连通裂缝模型; (c) 模型III-不连通裂缝模型
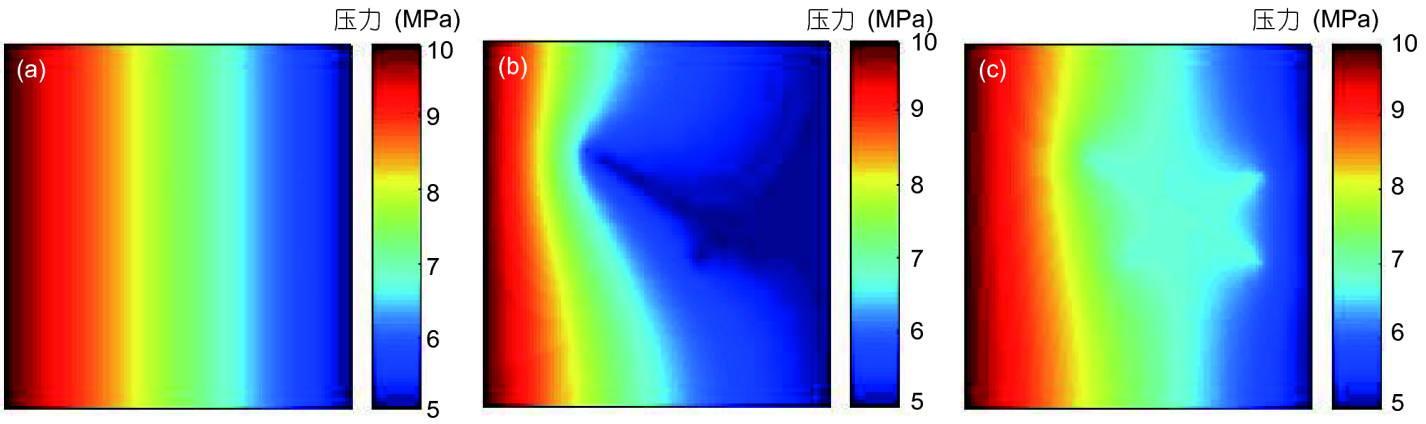

图 2 (网络版彩图)微尺度模型压力分布图. (a) 模型I; (b) 模型II; (c) 模型III

超过 $0.5 \%$ ，等效渗透率接近基质渗透率，且裂缝和流 出边界的连通情况对结果的影响也不明显.

当基质渗透率一定时, $k_{\mathrm{eq}} / k_{\mathrm{m}}$ 和裂缝开度关系曲线 如图3(b)所示. 当基质渗透率为 $0.001 \times 10^{-3} \mu \mathrm{m}^{2}$ 时, 随着 裂缝开度的增加, $k_{\mathrm{eq}} / k_{\mathrm{m}}$ 先保持不变, 然后逐渐增加, 最 后达到最大值并保持不变, 说明等效渗透率(模型渗流 能力)从一开始的由基质渗透率起主要作用逐渐转化 为由基质渗透率、裂缝开度和连通性共同影响，其中 最大值是由基质渗透率、裂缝开度和连通性共同决 定的.

微尺度模型研究表明: 当裂缝开度 $(0.4 \mu \mathrm{m})$ 相对较 小时, 裂缝以及裂缝和流出边界的连通性(连通、不连 通)对等效渗透率的贡献也相对较小, 等效渗透率主要 取决于基质渗透率的大小; 当裂缝开度相对足够大时 (基质渗透率为 $0.001 \times 10^{-3} \mu^{2}$ ), 裂缝和流出边界的连 通性对等效渗透率有重要影响。因此，裂缝对等效渗 透率的贡献率由基质渗透率、裂缝开度和连通性共同 作用.

\section{2 矿场尺度模型}

建立矿场尺度模型，利用可以模拟任意裂缝的 DFM方法研究基质渗透率在 $(0.0001 \sim 0.1) \times 10^{-3} \mu \mathrm{m}^{2}$, 裂 缝开度在0.1 1000 $\mu \mathrm{m}$ 之间变化时, 不同裂缝类型对生 产井的产量贡献率.

\subsection{1 模型假设}

由于本文研究对象为页岩气，所以模型中采用的 流体为可压缩单相流体 (只考虑甲烷气体)。假设流体 在裂缝系统属于一维流动, 在基质系统属于二维流动.

\subsection{2 渗流数学模型}

分别建立基质系统和裂缝系统页岩气渗流数学模 型. 裂缝被看作基质之间相交边界, 裂缝内部压力和基 质边界压力相等作为裂缝系统和基质系统的耦合 条件.

(1) 气体在基质中的渗流模型 

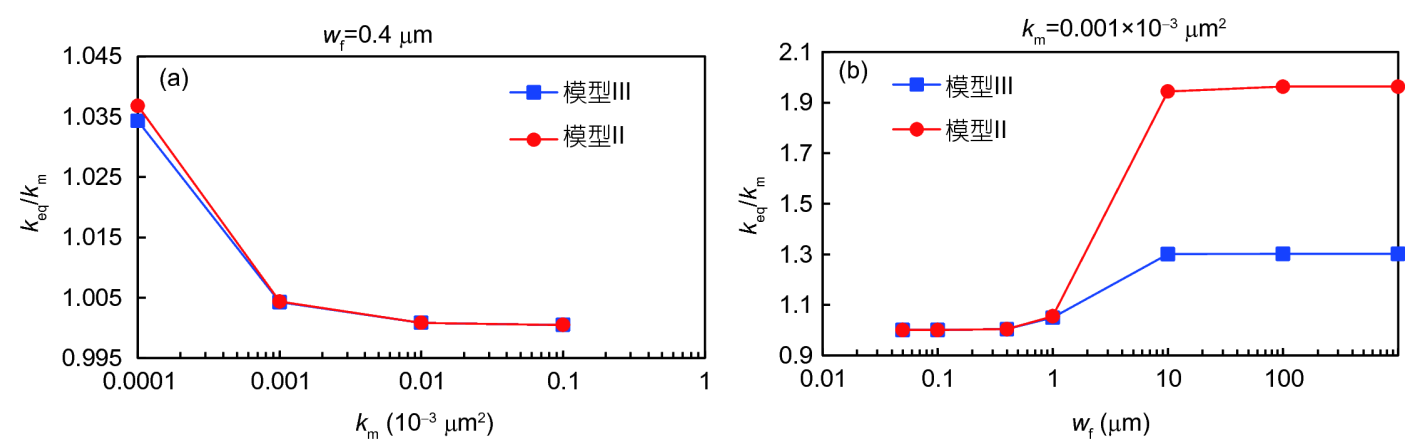

图 3 (网络版彩图) $k_{\mathrm{eq}} / k_{\mathrm{m}}$ 与基质渗透率和裂缝开度之间的关系. (a) 裂缝开度为 $0.4 \mu \mathrm{m}$ 时, $k_{\mathrm{eq}} / k_{\mathrm{m}}$ 和基质渗透率关系; (b) 基质渗 透率为 $0.001 \times 10^{-3} \mu \mathrm{m}^{2}$ 时, $k_{\mathrm{eq}} / k_{\mathrm{m}}$ 和裂缝开度关系

质量守恒方程可表示为

$\frac{\partial}{\partial t}\left(\rho \phi_{\mathrm{m}}\right)+\nabla \cdot\left(\rho u_{\mathrm{m}}\right)=Q_{\mathrm{m}}$,

其中, $\rho$ 为气体密度, 单位为 $\mathrm{kg} / \mathrm{m}^{3} ; \phi_{\mathrm{m}}$ 为基质孔隙度, 单 位为 $\% ; u_{\mathrm{m}}$ 为基质中的速度, 单位为 $\mathrm{m} / \mathrm{s} ; Q_{\mathrm{m}}$ 为源汇项, 单位为 $\mathrm{kg} /\left(\mathrm{m}^{3} \mathrm{~s}\right)$.

运动方程可表示为

$u_{\mathrm{m}}=-\frac{k_{\mathrm{m}}}{\mu_{\mathrm{g}}} \nabla p$,

其中, $k_{\mathrm{m}}$ 为基质渗透率, 单位为 $\times 10^{-3} \mu \mathrm{m}^{2} ; \mu_{\mathrm{g}}$ 为气体黏 度, 单位为 $\mathrm{mPa} \mathrm{s} ; p$ 为压力, 单位为 $\mathrm{MPa}$.

(2) 气体在裂缝中的渗流模型

质量守恒方程可表示为

$d_{\mathrm{f}} \frac{\partial}{\partial t}\left(\rho \phi_{\mathrm{f}}\right)+\nabla_{\mathrm{T}} \cdot\left(d_{\mathrm{f}} \rho u_{\mathrm{f}}\right)=d_{\mathrm{f}} Q_{\mathrm{m}}$,

其中, $d_{\mathrm{f}}$ 为裂缝厚度, 单位为 $\mu \mathrm{m} ; \phi_{\mathrm{f}}$ 为裂缝孔隙度, 单位 为 $\%$; $\nabla_{\mathrm{T}}$ 为裂缝切平面方向梯度因子; $u_{\mathrm{f}}$ 为裂缝中的速 度, 单位为 $\mathrm{m} / \mathrm{s}$.

由于裂缝厚度可以沿着裂缝改变, 所以式(3)两端 都有 $d_{\mathrm{f}}$ 项. 但是, 模型验证时裂缝厚度对最终结果不产 生影响, 假设所有裂缝均为垂直缝、厚度相同、且等 于储层厚度, 这样把三维模型降为二维, 所以式(3)简 化为

$\frac{\partial}{\partial t}\left(\rho \phi_{\mathrm{f}}\right)+\nabla_{\mathrm{T}} \cdot\left(\rho u_{\mathrm{f}}\right)=Q_{\mathrm{m}}$.

运动方程可表示为

$u_{\mathrm{f}}=-\frac{k_{\mathrm{f}}}{\mu} \nabla_{\mathrm{T}} p$.

\section{裂缝渗透率可表示为}

$k_{\mathrm{f}}=-10 \frac{w_{\mathrm{f}}^{2}}{12}$,

其中, $k_{\mathrm{f}}$ 为裂缝渗透率, 单位为 $\times 10^{-3} \mu \mathrm{m}^{2} ; w_{\mathrm{f}}$ 为裂缝开 度, 单位为 $\mu \mathrm{m}$.

联立方程(1) (6), 利用有限元法求解.

\section{2 .3 敏感性分析}

建立大小为 $1500 \mathrm{~m} \times 700 \mathrm{~m} \times 20 \mathrm{~m}$ 的单井模型(图 4): 其中模型I中只有 1 口水平井; 模型II中水平井周围 分布有 190 条天然裂缝; 模型III中通过20条主裂缝把水 平井和模型II中的天然裂缝连起来构成SRV; 模型IV又 在模型III的SRV内加入 30 条天然裂缝; 模型V在模型 III的SRV外加入 220 条天然裂缝.

所有模型均为封闭边界, 水平井定井底流压 $7 \mathrm{MPa}$ 生产, 初始地层压力为 $26 \mathrm{MPa}$, 基质孔隙度为 0.04 , 模型运行 10 年, 比较生产 10 年后不同模型之间累 产量变化关系. 其他参数相同情况下: 对比模型I和II研 究天然裂缝的贡献; 对比模型I和III研究SRV内人工裂 缝的贡献; 对比模型III和IV研究SRV内天然裂缝的贡 献; 对比模型III和V研究SRV外天然裂缝对总产量的 贡献.

当基质渗透率等于 $0.0001 \times 10^{-3} \mu \mathrm{m}^{2}$, 裂缝开度为 $100 \mu \mathrm{m}$, 生产到第 10 年时 5 类模型压力分布如图 5 所示. 对比发现: 裂缝-井筒连通情况直接决定压力的传播. 与模型I相比, 模型II虽裂缝发育, 但由于基质渗透率 低至 $0.0001 \times 10^{-3} \mu \mathrm{m}^{2}$, 压力传播主要由基质渗流能力 控制, 生产到第10年时没有传播到裂缝区; 与模型II相 

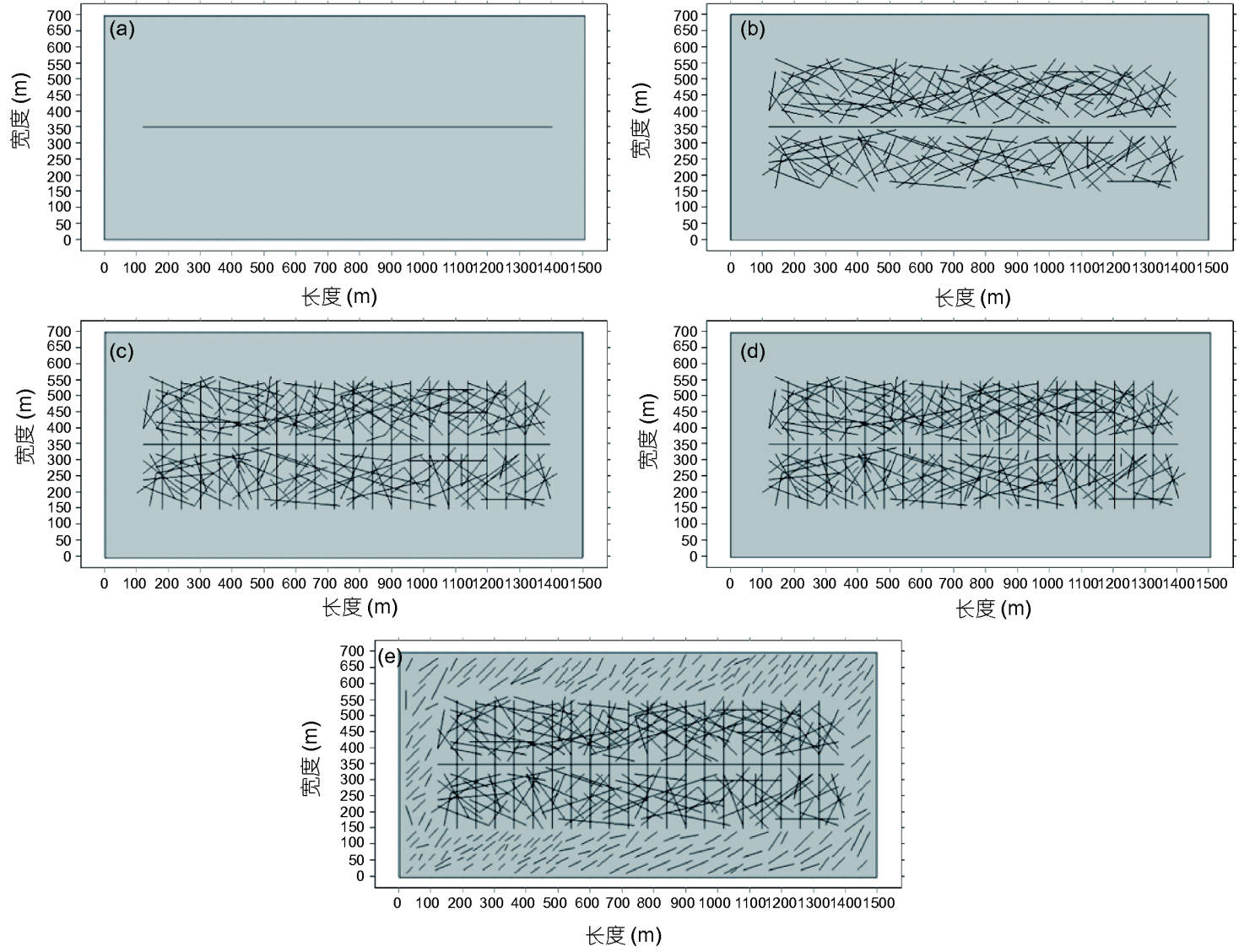

图 4 矿场尺度单井模型. (a) 模型I; (b) 模型II; (c) 模型III; (d) 模型IV; (e) 模型V

比，模型III中20条主裂缝沟通了模型II中的裂缝和井, 所以压力迅速传播到 SRV; 尽管模型IV和V分别在模 型III的SRV内和SRV外增加了大量裂缝，但由于这些 裂缝本身没有和SRV内与井连通的裂缝相连，且由于 基质渗透率较低，所以这些天然裂缝对压力的传播没 有明显贡献, 反而使计算复杂度增加了近 20 倍.

基质渗透率等于 $0.0001 \times 10^{-3} \mu \mathrm{m}^{2}$ ，裂缝开度取不 同值时 $(0.1,1,10,100,1000 \mu \mathrm{m})$, 模型 $\mathrm{V}$ 对应压力分 布如图6所示. 对比发现: 尽管模型V中存在SRV, 但由 于基质渗流能力本身较弱 $\left(0.0001 \times 10^{-3} \mu \mathrm{m}^{2}\right)$, 再加上裂 缝开度小于 $1 \mu \mathrm{m}$ 时, 裂缝渗透率低, 导流能力弱, 所以 压力波无法沿着连通的裂缝传播，如图6(a)和(b)所示; 如图6(c)所示, 当裂缝开度增加到 $10 \mu \mathrm{m}$ 时, 由于裂缝 渗透率及导流能力增强，压力波沿着人工裂缝迅速传 播; 如图6(d)所示, 当裂缝开度达到 $100 \mu \mathrm{m}$ 时，压力传 播愈加明显; 但是, 当裂缝开度继续增加到 $1000 \mu \mathrm{m}$ 时,
由于此时压力传播主要受基质渗透率 $\left(0.0001 \times 10^{-3}\right.$ $\mu \mathrm{m}^{2}$ ) 限制, 且SRV外天然裂缝没有连通, 生产到 10 年时, 压力波并没有明显继续向外传播到气藏边界，如 图6(e)所示.

裂缝开度为 $100 \mu \mathrm{m}$ ，基质渗透率等于 $(0.0001$, $0.001,0.01,0.1) \times 10^{-3} \mu \mathrm{m}^{2}$, 模型 $\mathrm{V}$ 对应压力分布如图7 所示. 当基质渗透率为 $0.0001 \times 10^{-3} \mu \mathrm{m}^{2}$, 由于压力波从 SRV内传播到SRV外的过程主要由渗流能力较弱的基 质控制, 所以外区天然裂缝的影响不明显, 压力无法传 播到边界处, 如图7(a)所示; 如图7(b)所示, 当基质渗透 率增大到 $0.001 \times 10^{-3} \mu \mathrm{m}^{2}$ 时，由于基质渗流能力增加， SRV区内基质压力继续降低，且压力波逐渐向 SRV外 区传播，人工裂缝促使压力向外传播，裂缝的不均匀 分布引起了压力波前缘不均匀推进; 如图7(c)所示，当 基质渗透率达到 $0.01 \times 10^{-3} \mu^{2}$, 生产到第 10 年时, 压力 波已传播到气藏边界; 如图7(d)所示, 当基质渗透率等 

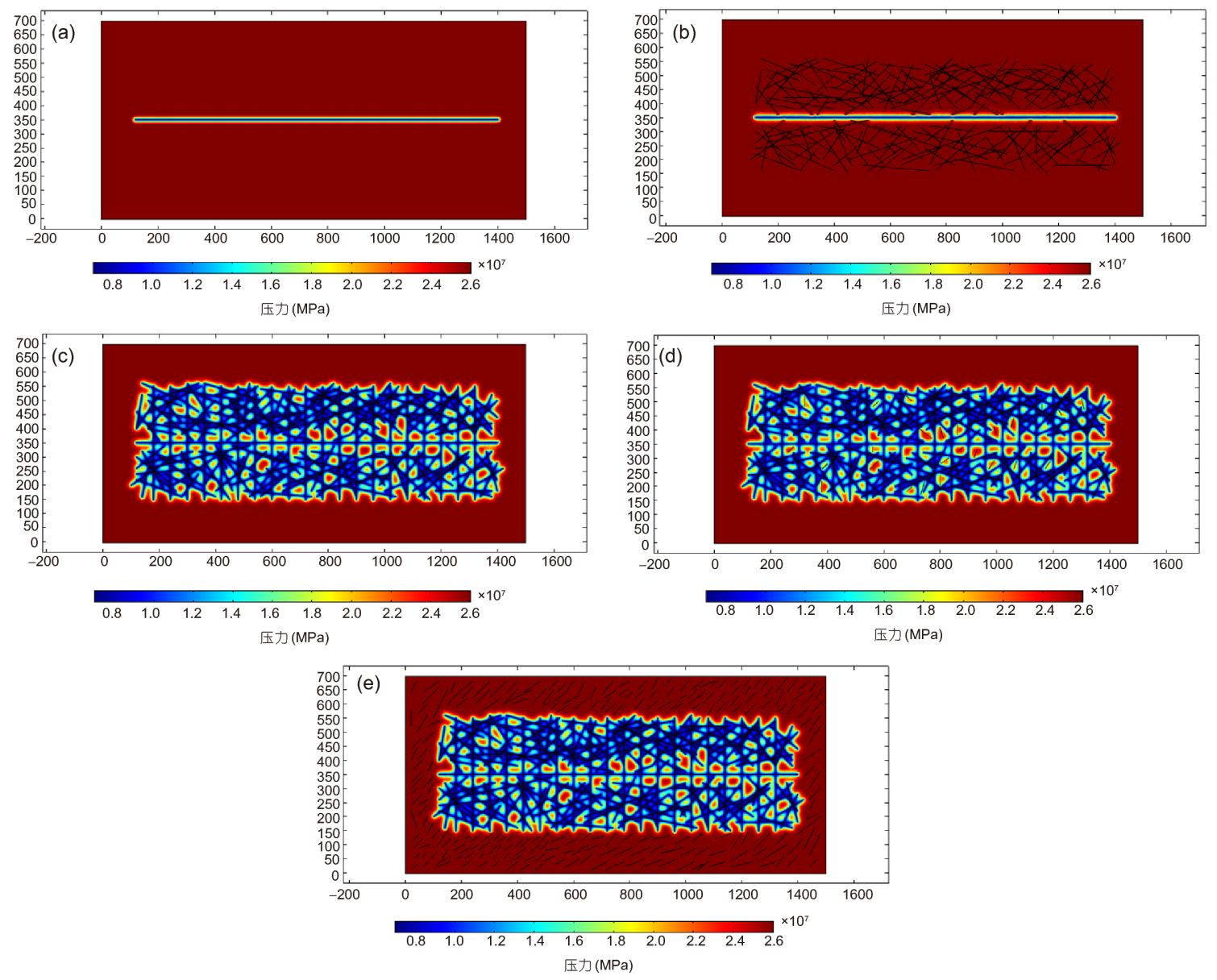

图 5 (网络版彩图)不同模型压力分布图(10年). (a) 模型I; (b) 模型II; (c) 模型III; (d) 模型IV; (e) 模型V

于 $0.1 \times 10^{-3} \mu \mathrm{m}^{2}$ ，生产到第 10 年时，整个气藏压力接近 井底流压.

综合分析不同模型生产 10 年时，不同级别基质渗 透率、裂缝开度对应的压力分布结果发现，压力传播 快慢在受裂缝连通性、裂缝开度、基质渗透率绝对大 小影响的同时其相对大小关系也很重要. 为了进一步 量化其作用强弱，指导页岩气藏选取有效的数值模拟 方法，定量计算不同级别基质渗透率、裂缝开度页岩 气藏生产 10 年时，不同裂缝类型对页岩气井总产量的 贡献率.

同时，5种模型算法复杂度如图8所示，模型I所需 时间和内存最少, 模型 $\mathrm{V}$ 消耗时间和内存空间最多. 总 体上说，算法复杂度和裂缝数量呈正相关，裂缝越多， 网格数量越多, 算法复杂度越高.
其他参数完全相同情况下(除模型II中存在天然裂 缝), 生产 10 年时模型II比模型I的产量增量百分比如图 9(a)所示: (1) 当基质渗透率大于 $0.1 \times 10^{-3} \mu \mathrm{m}^{2}$, 裂缝开 度小于 $10 \mu \mathrm{m}$; (2) 当基质渗透率 $0.01 \times 10^{-3} \mu \mathrm{m}^{2}<k_{\mathrm{m}}<$ $0.1 \times 10^{-3} \mu \mathrm{m}^{2}$, 裂缝开度小于 $1 \mu \mathrm{m}$; 与模型I相比，模型 II生产 10 年时总产量提高不足 $4.09 \%$. 因此，如果可接 受工程误差不超过 $5 \%$, 对于模型II来说, 当基质渗透率 和裂缝开度满足条件(1)和(2)时, 天然裂缝对总产量的 贡献较小. 所以，综合考虑天然裂缝引起的计算复杂 度和对计算精度的提高程度, 模型II在(1)和(2)条件下 模拟时，可通过构建表观渗透率和连续介质模型结合 的方法表征天然裂缝; 否则, 需采用离散介质模型. 但 $\mathrm{SRV}$ 是页岩气藏商业生产的前提, 因此, 页岩气藏生产 时一般不会出现模型II的情况. 

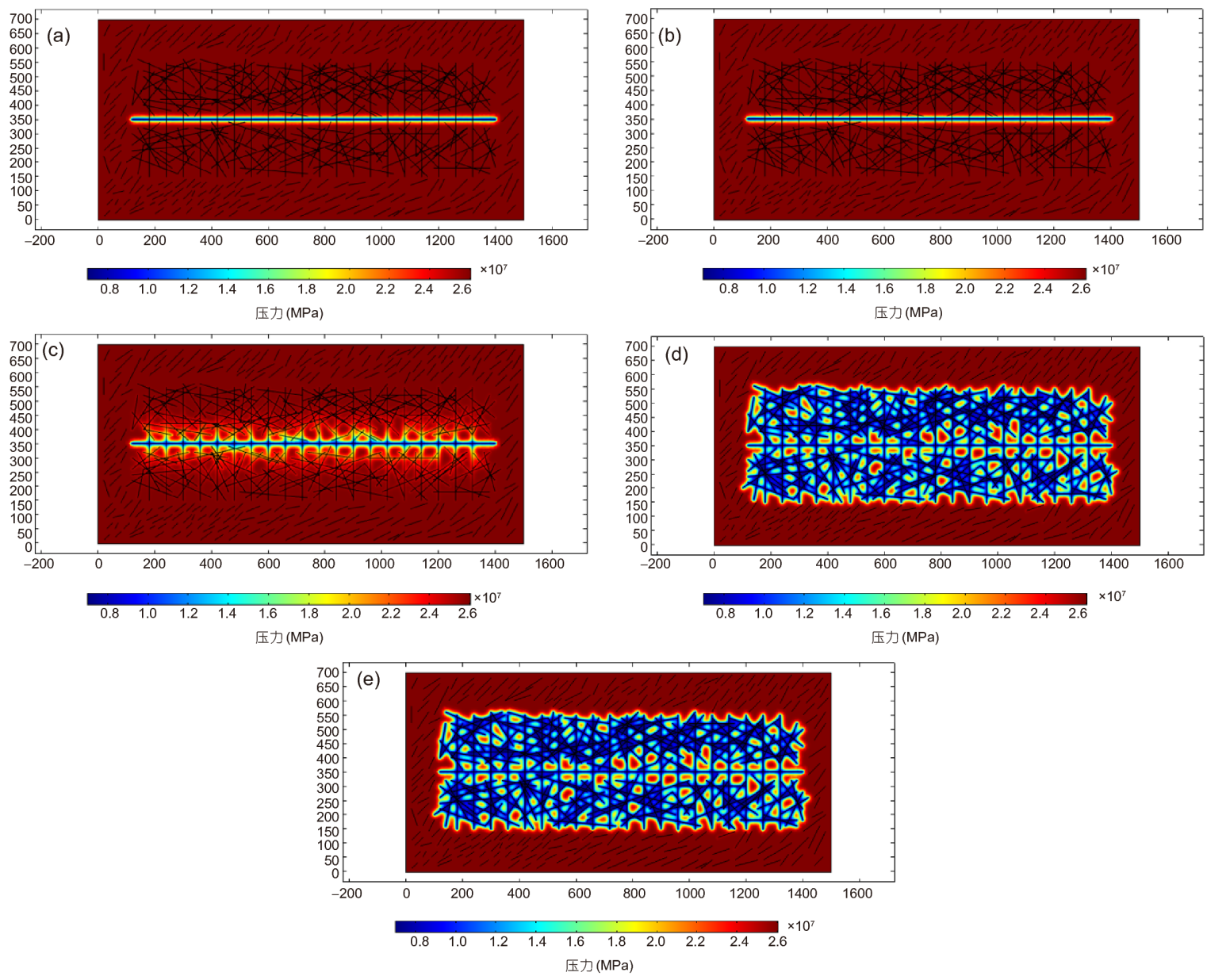

图 6 (网络版彩图)裂缝开度不同时模型 $\mathrm{V}$ 压力分布图(10年). (a) $w_{\mathrm{f}}=0.1 \mu \mathrm{m}$; (b) $w_{\mathrm{f}}=1 \mu \mathrm{m}$; (c) $w_{\mathrm{f}}=10 \mu \mathrm{m}$; (d) $w_{\mathrm{f}}=100 \mu \mathrm{m}$; (e) $w_{\mathrm{f}}$ $=1000 \mu \mathrm{m}$

其他参数完全相同情况下(除了模型III中存在人 工裂缝), 生产 10 年时模型III比模型I的产量增量百分 比如图9(b)所示: (1) 当基质渗透率大于 $0.1 \times 10^{-3} \mu \mathrm{m}^{2}$, 裂缝开度小于 $100 \mu \mathrm{m}$ 时; (2) 当基质渗透率满足 $0.01 \times 10^{-3} \mu \mathrm{m}^{2}<k_{\mathrm{m}}<0.1 \times 10^{-3} \mu \mathrm{m}^{2}$ ，裂缝开度小于 $1 \mu \mathrm{m}$ 时；与模型I相比，模型III生产 10 年时总产量提高不足 $4.35 \%$. 因此, 如果可接受工程误差不超过 $5 \%$, 对于模 型III来说，当基质渗透率和裂缝开度满足条件(1)和 (2)时, 人工裂缝对总产量的贡献较小. 所以, 综合考虑 人工裂缝引起的计算复杂度和对计算精度的提高程 度, 模型III在(1)和(2)条件下进行数值模拟时, 连续介质 模型方法就可以等效处理这些人工裂缝，而不需要采 用离散介质方法; 否则, 要采用离散介质模型. 显然页
岩气藏基质渗透率和裂缝开度不满足条件(1)和(2), 因 此, 模拟页岩气藏人工裂缝时需采用离散介质模型.

在其他参数完全相同的情况下(除模型IV的SRV 内存在天然裂缝), 生产 10 年时模型IV比模型III的产量 增量百分比如图 9(c) 所示: 当基质渗透率大于 $0.01 \times 10^{-3} \mu \mathrm{m}^{2}$, 裂缝开度小于 $1000 \mu \mathrm{m}$ 时，生产 10 年时 模型IV比模型III的总产量提高不足 $4.35 \%$; 如果基质 渗透率小于 $0.01 \times 10^{-3} \mu \mathrm{m}^{2}$, 天然裂缝贡献率将更低. 因 此, 如果可以接受工程误差不超过 $5 \%$, 在页岩气基质 渗透率范围内, SRV内天然裂缝对总产量贡献较小. 综 合考虑SRV内天然裂缝引起的计算复杂度和对计算精 度的提高程度, 进行页岩气藏数值模拟时, 可通过构建 表观渗透率和连续介质模型结合的方法处理SRV内天 

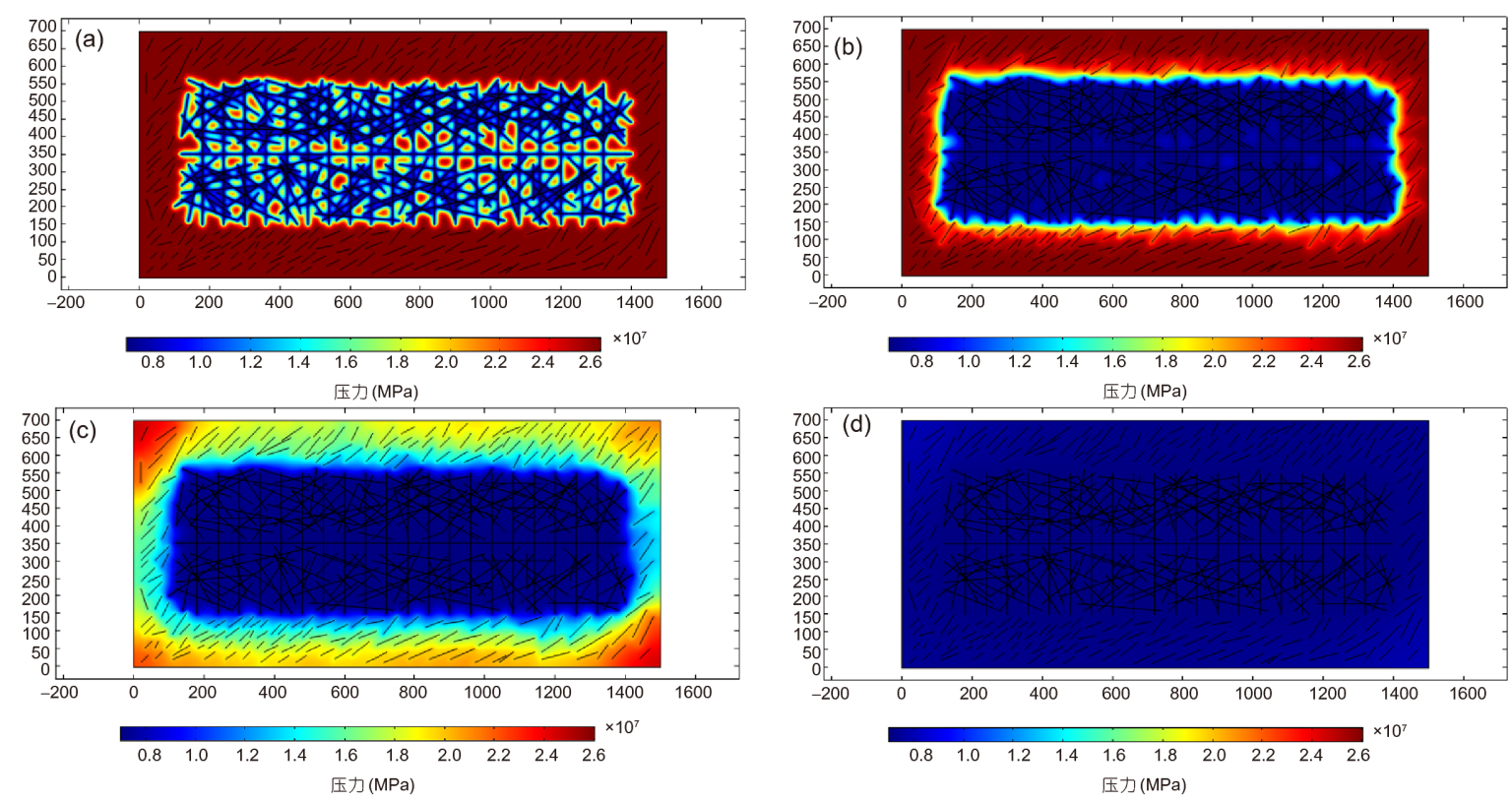

图 7 (网络版彩图)基质渗透率不同时模型 $\mathrm{V}$ 压力分布图(10年). (a) $k_{\mathrm{m}}=0.0001 \times 10^{-3} \mu \mathrm{m}^{2} ;$ (b) $k_{\mathrm{m}}=0.001 \times 10^{-3} \mu \mathrm{m}^{2} ;(\mathrm{c}) k_{\mathrm{m}}=0.01$ $\times 10^{-3} \mu \mathrm{m}^{2}$; (d) $k_{\mathrm{m}}=0.1 \times 10^{-3} \mu \mathrm{m}^{2}$

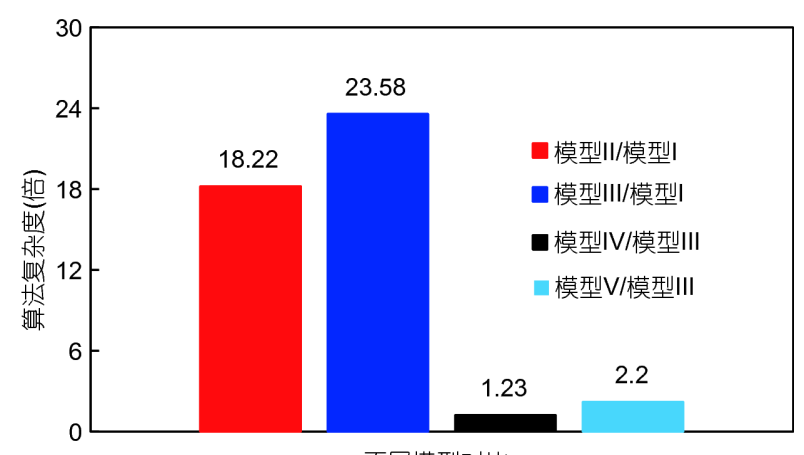

不同模型对比

然裂缝, 而没必要采用离散介质模型.

其他参数完全相同(除了模型 $\mathrm{V}$ 的SRV 外存在天然 裂缝), 生产 10 年时模型 $\mathrm{V}$ 比模型III的产量增量百分比 如图9(d)所示: 当基质渗透率大于 $0.0001 \times 10^{-3} \mu \mathrm{m}^{2}$, 裂 缝开度小于 $1000 \mu \mathrm{m}$, 生产 10 年时模型 $\mathrm{V}$ 比模型III的总 产量提高不足 $4.20 \%$; 如果基质渗透率小于 $0.0001 \times$ $10^{-3} \mu \mathrm{m}^{2}$, 裂缝几乎不起作用, SRV外天然裂缝贡献率 将更低. 因此, 如果可接受工程误差不超过 $5 \%$, 在页岩 气基质渗透率范围内, SRV外天然裂缝对总产量的贡 献相对较小. 综合考虑SRV外天然裂缝引起的计算复

杂度和对计算精度的提高程度, 进行页岩气藏数值模 拟时, 可通过构建表观渗透率和连续介质模型相结合 的方法处理SRV外天然裂缝, 不需要采用离散介质 模型.

矿场尺度模型研究表明: 裂缝性储层中流体的流 动能力由基质渗透率、裂缝连通性以及裂缝开度等共 同决定. 在页岩气藏基质渗透率范围内, SRV内人工裂 缝对页岩气产量起主要作用, 需通过离散介质模型表 征; SRV内和SRV外天然裂缝对产量的贡献相对较低, 可通过构建表观渗透率 ${ }^{[6,27]}$ 的方法简化表征.

\section{4 结论}

(1) 裂缝对流体流动能力的影响由基质渗透 率、裂缝导流能力(裂缝开度)以及裂缝连通性等共同 决定.

(2) 人工压裂形成SRV是页岩气藏实现商业开发 的前提. 微尺度模型和矿场尺度模型研究表明: 在页 岩气藏基质渗透率范围内, 且存在SRV改造区的条件 下, 页岩气产量以SRV内人工裂缝的贡献为主, SRV内 以及SRV外天然裂缝的贡献量相对较小.

（3）综合考虑计算复杂度和计算精度, 通过构建基 


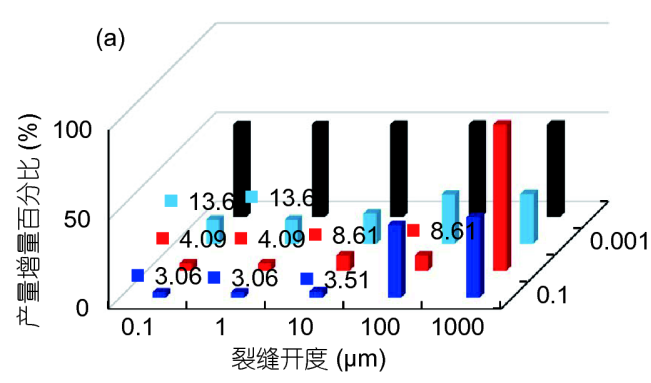

(c)

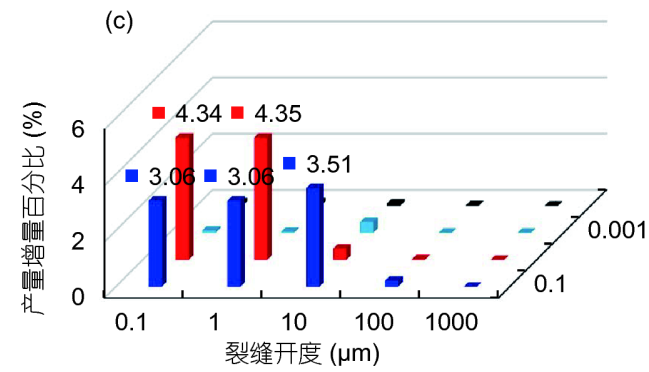

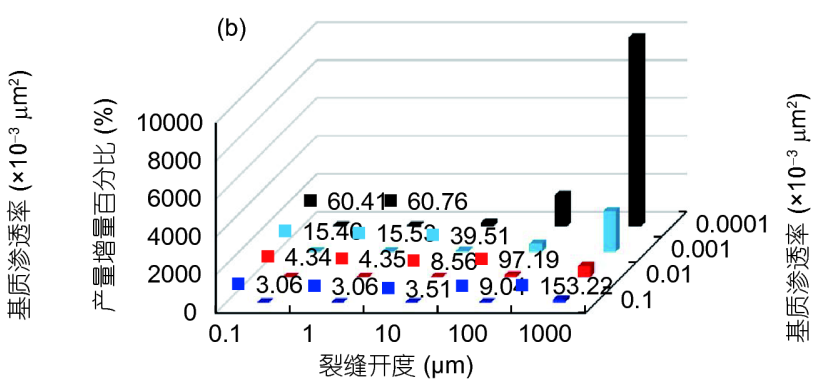

(d)

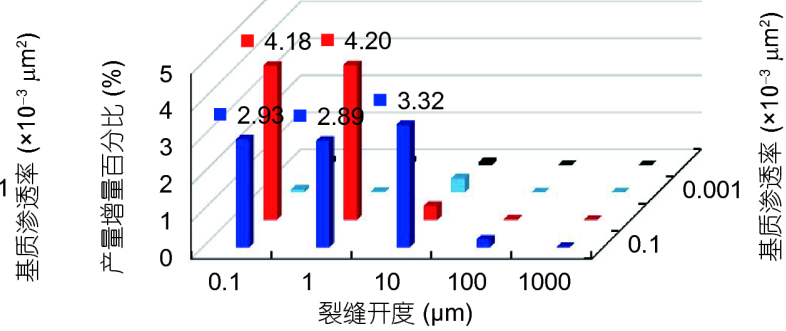

图 9 (网络版彩图)模型产量增量的百分比. (a) 模型II比模型I的产量增量百分比; (b) 模型III比模型I的产量增量百分比; (c) 模 型IV比模型III的产量增量百分比; (d) 模型V比模型III的产量增量百分比

质-天然裂缝表观渗透率和连续介质模型的方法表征 天然裂缝，采用离散介质模型中计算网格少、效率高
的DFN模型表征人工裂缝是高效离散裂缝网络页岩气 藏多尺度渗流数值模拟的合理处理方法.

\section{参考文献}

1 Warren J E, Root P J. The behavior of naturally fractured reservoirs. SPE J, 1963, 3: 245-255

2 Mi L D, Jiang H Q, Li J J, et al. The investigation of fracture aperture effect on shale gas transport using discrete fracture model. J Nat Gas Sci Eng, 2014, 21: 631-635

3 糜利栋, 姜汉桥, 李涛, 等. 基于离散裂缝模型的页岩气动态特征分析. 中国石油大学学报(自然科学版), 2015, 39: 126-131

4 Sun J, Schechter D. Optimization-based unstructured meshing algorithms for simulation of hydraulically and naturally fractured reservoirs with variable distribution of fracture aperture, spacing, length, and strike. SPE Reserv Eval Eng, 2015, 18: 463-480

5 Mi L D, Jiang H Q, Li J J. The impact of diffusion type on multiscale discrete fracture model numerical simulation for shale gas. J Nat Gas Sci Eng, 2014, 20: 74-81

6 Sun J, Schechter D. Investigating the effect of improved fracture conductivity on production performance of hydraulically fractured wells: Fieldcase studies and numerical simulations. J Can Pet Tech, 2015, 54: 442-449

7 McKoy M L, Sams W N. Tight gas reservoir simulation: Modeling discrete irregular strata-bound fracture networks and network flow, including dynamic recharge from the matrix. In: Proceedings of the Natural Gas Conference Emerging Technologies for the Natural Gas Industry. Washington DC: US Department of Energy's Federal Energy Technology Center Publication, 2010

8 Boyle E J, Sams W N. NFFLOW: A reservoir simulator incorporating explicit fractures. In: SPE Western Regional Meeting. Bakersfield, 2012

9 Li J J, Jiang H, Jiang L, et al. Varying-scale shale gas flow: Discrete fracture networks (DFN) based numerical simulation. Particul Sci Tech, 2016, 34: 557-564

10 Mi L D, Jiang H Q, Mou S B, et al. Numerical simulation study of shale gas reservoir with stress-dependent fracture conductivity using multiscale discrete fracture network model. Particul Sci Tech, 2018, 36: 202-211

11 Mi L D, Yan B C, Jiang H Q, et al. An Enhanced Discrete Fracture Network model to simulate complex fracture distribution. J Pet Sci Eng, 2017, 156: $484-496$ 
12 Loucks R G, Reed R M, Ruppel S C, et al. Morphology, genesis, and distribution of nanometer-scale pores in siliceous mudstones of the Mississippian Barnett Shale. J Sediment Res, 2009, 79: 848-861

13 邹才能, 李建忠, 董大忠, 等. 中国首次在页岩气储集层中发现丰富的纳米级孔隙. 石油勘探与开发, 2010, 5: 4-5

14 邹才能, 董大忠, 王社教, 等. 中国页岩气形成机理, 地质特征及资源潜力. 石油勘探与开发, 2010, 37: 641-653

15 聂海宽, 张金川. 页岩气储层类型和特征研究——以四川盆地及其周缘下古生界为例. 石油实验地质, 2011, 33: 219-225

16 Javadpour F, Fisher D, Unsworth M. Nanoscale gas flow in shale gas sediments. J Canad Pet Tech, 2007, 46: 55-61

17 Wu K, Li X, Wang C, et al. A model for gas transport in microfractures of shale and tight gas reservoirs. AIChE J, 2015, 61: 2079-2088

18 薛冰, 张金川, 唐玄, 等. 黔西北龙马溪组页岩微观孔隙结构及储气特征. 石油学报, 2015, 36: 138-149

19 Gale J F W, Reed R M, Holder J. Natural fractures in the Barnett Shale and their importance for hydraulic fracture treatments. Bulletin, 2007, 91 : 603-622

20 Gale J F W, Laubach S E, Olson J E, et al. Natural fractures in shale: A review and new observations. Bulletin, 2014, 98: 2165-2216

21 糜利栋. 离散裂缝网络页岩气藏多尺度渗流数值模拟方法研究. 博士学位论文. 北京: 中国石油大学(北京), 2017

22 Moinfar A. Development of an efficient embedded discrete fracture model for 3D compositional reservoir simulation in fractured reservoirs. Dissertation for Doctoral Degree. Austin: The University of Texas at Austin, 2013

23 Moinfar A, Varavei A, Sepehrnoori K, et al. Development of an efficient embedded discrete fracture model for 3D compositional reservoir simulation in fractured reservoirs. SPE J, 2014, 19: 289-303

24 Cao Y, Killough J E. An improved boundary element method for modeling fluid flow through fractured porous medium. In: SPE Reservoir Simulation Conference. Montgomery, 2017

25 Durlofsky L J. Upscaling of geocellular models for reservoir flow simulation: A review of recent progress. In: 7th International Forum on Reservoir Simulation. Baden-Baden, 2003. 23-27

26 糜利栋, 姜汉桥, 姜俊键. 耦合基质-天然裂缝渗流机理的页岩气表观渗透率. 中国科学: 技术科学, 2016, 46: 1064-1070

27 Mi L D, Jiang H Q, Li J J, et al. A novel permeability model of naturally fractured shale reservoirs. In: SPE Russian Petroleum Technology Conference and Exhibition. Moscow, 2016. 24-26 


\title{
Evaluation and selection of numerical simulation methods for shale gas reservoirs
}

\author{
MI LiDong ${ }^{1,2,3}$, JIANG HanQiao $^{3}$, HU XiangYang ${ }^{1,2}$, LI JunJian ${ }^{3} \&$ JIA Ying ${ }^{1,2}$ \\ ${ }^{1}$ Sinopec Petroleum Exploration and Production Research Institute, Beijing 100083, China; \\ ${ }^{2}$ Sinopec Key Laboratory of Marine Oil and Gas Reservoirs, Beijing 100083, China; \\ ${ }^{3}$ College of Petroleum Engineering, China University of Petroleum (Beijing), Beijing 102249, China
}

The complex fracture network brings great challenge to the numerical simulation of shale gas reservoirs. Based on the discrete fracture model (DFM), micro scale model and field scale model are established to investigate the influence of fracture types on the reservoir performance when the correspondence of matrix permeability and fracture aperture is different. Results show that: because the magnitude of shale gas reservoir matrix permeability is $\left(10^{-8}-10^{-4}\right) \times 10^{-3} \mu \mathrm{m}^{2}$ and the SRV is a prerequisite for shale gas production, hydraulic fractures play an important role in the shale gas production while the contribution of natural fractures in and out the SRV is relatively small. Therefore, considering the computational complexity and precision, natural fractures can be characterized by matrix-natural fracture apparent permeability model and continuum model while the hydraulic fracture can be characterized by the discrete fracture network (DFN) with less computational grids and high efficient.

natrual fracture, hydraulic fracture, stimulate reservoir volume, shale gas reservoir, numerical simulation method

doi: $10.1360 / \mathrm{N} 092017-00152$ 